\title{
Design of Resistance Measurement Equipment for Motor Commutator Segment Based on LabWindows/CVI
}

\author{
Wang Yong ${ }^{1, *}$, Wang Xiaohong ${ }^{2}$, Zhang Liwen ${ }^{1}$, Zhang Jincan ${ }^{1}$ and Tian Wei ${ }^{1}$ \\ ${ }^{1}$ College of Electrical Engineering, Henan University of Science and Technology, Luoyang, 471023, China \\ ${ }^{2}$ College of Information Engineering, Henan University of Science and Technology, Luoyang, 471023, China
}

\begin{abstract}
Traditional resistance measurement for DC motor commutator segment is manual. The process is complicated, the efficiency is low, and the cost is high. In order to enhance efficiency and precision of the resistance measurement, we design an automatic measurement equipment using virtual instrument technique. The equipment consists on industrial compute, stepping motor drive transmission system, low resistance measuring meter, host compute software. Making the equipment universal and automated is the core of design. We discuss working principle of the equipment, hardware design, software design, measurement experiment process and results analysis. Hardware design includes accurate position control of stepping motor, 4-wire method for resistance measurement, special fixture design for probe. Software design based on LabWindows/CVI platform includes serial communication, asynchronous timers, multi-thread, ActiveX control. The measurement experiment results indicate that the euipment can measure almost all kind of DC motor rotors accurately, also can record and save measurement results automatically.
\end{abstract}

Keywords: Commutator segment, Low resistance measuring meter, LabWindows/CVI, Multi-thread

\section{INTRODUCTION}

When DC motor runs, faults about armature winding such as wire break, open weld, commutator segment break, short circuit between segments, and so on, can cause electromagnetic asymmetry to produce commutation spark [1]. Measuring resistance of between two adjacent commutator segments can determine whether there are the above faults in a motor rotor. As a basic rule, conformity errors of the resistance values between the two adjacent commutator segments should be not more than 5\%. Therefore, measuring resistance of between two adjacent commutator segments is a constant and necessary quality test item of DC motor. Our client motor manufacturer produces many different types of DC motor, and the number of DC motor's commutator segment varys from 5 to 120 , and range of resistance value between two adjacent segments is also different. Traditional manual measurement can bring more complicated process, lower efficiency, and higher cost. And some segments' measurement may be missing. In order to enhance efficiency and precision of resistance measurement for DC motor commutating segment, we have designed an automatic measurement equipment based on LabWindows/CVI software $[2,3]$.

\section{COMPOSITION AND PRINCIPLE OF SYSTEM}

The diameter and the commutator segment number of every motor rotor, range of resistance value between two adjacent segments are different. The performance of automatic measurement equipment we have designed is required to be universal. It can measure most of DC motor rotors, and can automatically record and save measurement results. As shown in Fig. (1), in order to meet the above measurement requirements, the automatic measurement equipment is mainly composed of industrial compute, stepping motor drive transmission system, low resistance measuring meter, host compute software. Industrial compute is the main control unit of the whole system. Host compute software is programed to control two RS232 serial ports. One of ports is used to connect stepping motor drive transmission system to determine the touched position of commutator segment and test probe, and the angle of motor rotor rotation every time. The other serial port is used to set parameters of low resistance measuring meter and read resistance values being measured back. Fixture is used to fix the probes being used to touch measured commutator segments. It can make sure the probes and measured commutator segments have close contact $[4,5]$.

\section{HARDWARE DESIGN}

\subsection{Drive Transmission System of Stepping Motor}

The drive transmission system is used to control the stepping motor which is installed within the equipment cabinet and has a reducing gear. The measured motor rotor is fixed in the chuck which mounts on the stepping motor's shaft. When industrial compute sends instructions to control stepmotor system to run, the measured rotor turns same angle with stepping motor. The turning angle can be adjusted through software's calculation according to number of every 


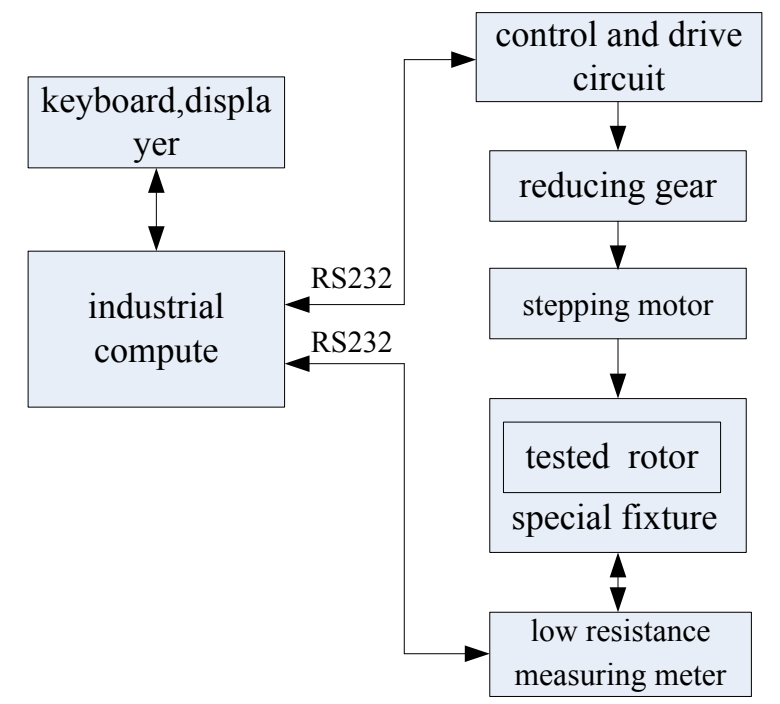

Fig. (1). Structure diagram of the measurement equipment.

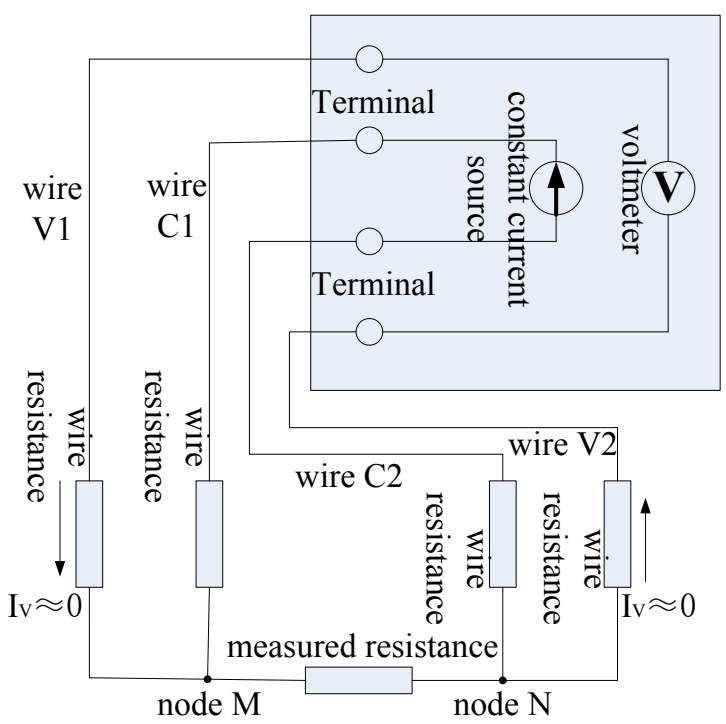

Fig. (2). Working principle of measuring resistance with four-wire.

measured rotor's commutator segments, so two probes being used to measure can be pinpoint precisely on the two adjacent measured commutator segments. The expression (1) is used to calculate accurately the turning angle.

Step $=\frac{360^{\circ} \times M C S \times N}{\text { Num } \times \text { Angle }}$

Where Step is number of running step of stepping motor every time, $M C S$ is subdividing value of stepping motor, Num is number of measured rotor's commutator segments, Angle is the turning angle of stepping motor's every step.

\subsection{Low Resistance Measuring Meter}

Low resistance measuring meter is used to measure resistance between two adjacent commutator segments and send measuring results to industrial compute through its programmable interface. Different type motors have different commutator segment resistance values. Their range is $20 \mathrm{~m} \Omega$ $\sim 1.5 \mathrm{~K} \Omega$. Because the smallest measured resistance's value is milliohm level which can be compared with resistance of connecting wire. So conventional resistance measuring meter, which adopt a method of measuring resistance with twowire, cann't be used. Low resistance measuring meter used in the equipment adopts a method of measuring resistance with four-wire, its measuring range is $3 \mathrm{~m} \Omega \sim 30 \mathrm{~K} \Omega$. Its output current range from internal constant current source is $10 \mathrm{~A} \sim 10 \mu \mathrm{A}$, and is divided into 5 class. Its working principle is as shown in Fig. (2).

In the method of measuring resistance with four-wire, one pair of wires (wire $\mathrm{C} 1$ and $\mathrm{C} 2$ ) connects the internal constant current source, the other pair of wires(wire V1 and 


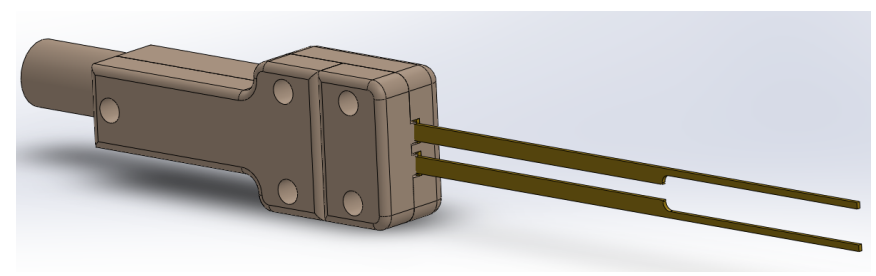

Fig. (3). Fixture for Probe.

V2) connects the internal voltmeter [5-7]. The constant current source loop and voltmeter loop are separate. So output current from the internal constant current source isn't affected by resistance of connecting cable and measured resistance. And because input impedance $(10 \mathrm{M} \Omega)$ of the selected voltmeter is much larger than measured resistance, the current equation about node M in Fig. (2) is expression (2).

$I_{L}=I_{R}+I_{V}, I_{V} \approx 0 \Rightarrow I_{L} \approx I_{R}$

Where $I_{L}$ is the current which flows through measured resistance, $I_{R}$ is the current which flows through wire $C 1, I_{V}$ is the current which flows through wire V1. That is, output current from constant current source only flows through the measured resistanc, don't divert to terminals of voltmeter [811]. So

$\mathrm{V}=U_{\llcorner}+2 U_{\odot} \approx U_{\perp}=\mathrm{I}_{\llcorner} \times \mathrm{R}_{\llcorner} \Rightarrow \mathrm{R}_{\llcorner} \approx \mathrm{V} / \mathrm{I}_{\perp}$

Where $V$ is voltage value measured by the voltmeter, $U_{L}$ is voltage value across the measured resistor, $U$ is voltage value across the connecting wire V1(or wire V2), $I_{L}$ is the current through measured resistance. Therefore, as long as the two loops in the method of measuring resistance with four-wire to intersect at two nodes (node $\mathrm{M}$ and node $\mathrm{N}$ in Fig. 2), negative effect from connecting cable can be suppressed, and the accuracy of the measurement results can be ensured.

\subsection{Fixture Design for Probe}

Because the commutator segments on rotors cann't be disassembled and cann't have any damage during the measuring process, so the low resistance measuring meter own's alligator clips cann't clamp the commutator segments directly. Instead, the method using copper probe to contact surface of commutator segments with pressure is adopted. In order to follow the principle of measuring resistance with four-wire in the process of press-connection measurement, the original alligator clips are cut off, the remaining two wires connected with the alligator clips originally are welded separately on the two copper probes which are fixed in specially-made fixture with bakelite, as shown in Fig. (3). The two probes contact closely on surface of the measured commutator segments by moving the fixture. That is comparable to that wire $\mathrm{V} 1$ and wire $\mathrm{C} 1$ meet in node $\mathrm{M}$ in Fig. (2). In order to operate easily and make sure the probes contact closely with the measured commutator segments, the fixtures are mounted on the mechanical magnetic stands. Before start measurement, the magnetic stands are moved to determine position of the probes, then they are locked.

\section{SOFTWARE DESIGN}

\subsection{System Framework Design of Software}

The operating interface software is developed based on LabWindows/CVI produced by NI company. The main functions of the operating interface software cover: (1) measure accurately resistances and read result back; (2) control stepping motor drive transmission system to run to drive the measured rotor to rotate the precise angle; (3) Pause or continue the measurement at any moment, and support the equipment scram. (4) Cut off the power supply of the equipment after a long stopping time. (5) Record measuring datas and save them in Word format files. The design flow chart of the operating interface software is as shown in Fig. (4).

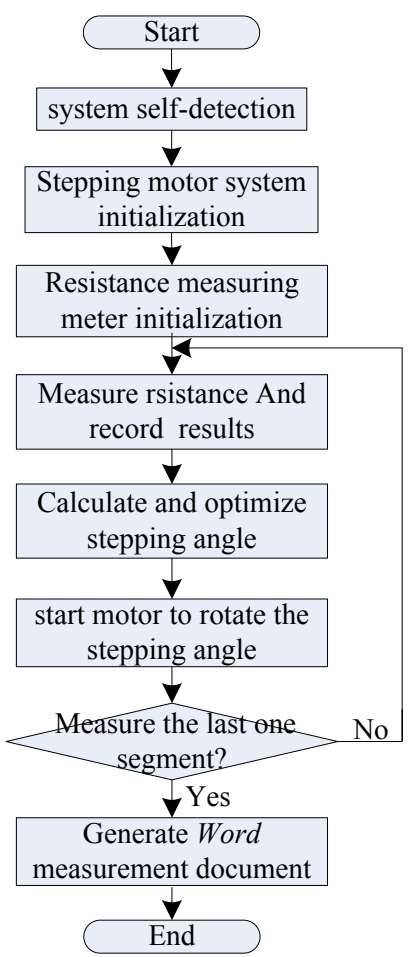

Fig. (4). Design Flow Chart of Operating Software.

\subsection{Function Realization of Operating Interface Soft- ware}

The main user interface is as shown in Fig. (5). The key technologys about serial communication, asynchronism 


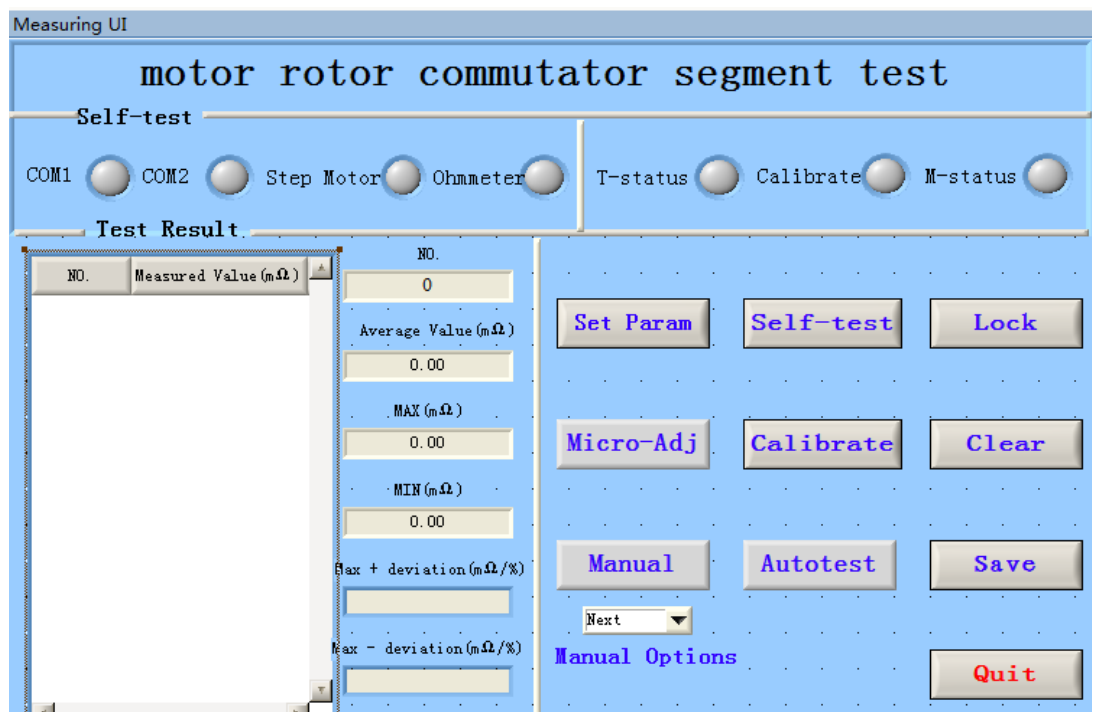

Fig. (5). Main User Interface of Operating Software.

timer, multithreading, ActiveX controls, etc are applied in the UI software.

\subsubsection{Design of Stepping Motor Drive Transmission Sys- tem and Low Resistance Measuring Meter}

The UI software control the stepping motor drive transmission system and the low resistance measuring meter to work via two serial ports. The design keypoints are to calculate the turning angle of stepping motor's every step and to set measuring range, sampling rate, triggering mode, etc of the low resistance measuring meter. The main program is as follows:

$/ *$ Calculations program about the stepping motor running steps every single measurement $* /$

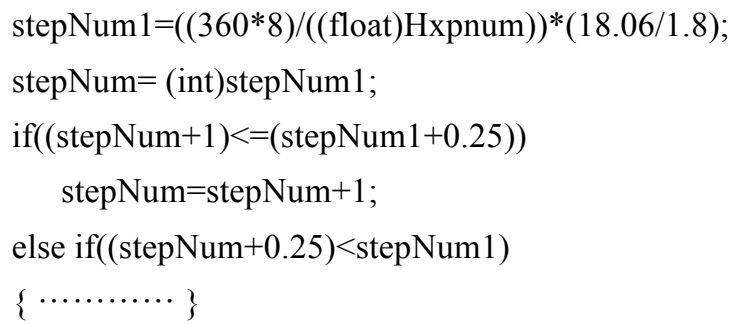

ComStepSend(stepNumChar);// send instruction about running steps

/*Set parameters of the low resistance measuring meter*/

ComOhmSend("term a");// select Port

ComOhmSend(":samp:rate fast"); // set sampling rate

ComOhmSend("res:rang:auto on");// set measuring range ComOhmSend(":trig:sour imm");// set triggering mode

\subsubsection{Design of Pause and Scram}

Every rotor on some motors has 120 commutator segments, so it need take some time to complete measurement for the whole rotor. In order to make operation simpler and more human-friendly, pause or continuing function at any moment during measuring process is designed in UI. During measuring process, when some electrical faults such as short-circuit etc. happen, the measurement equipment need stop immediately the current running to protect electronic parts. So the function of controlling the equipment to scram also need be designed in UI.

A single-threaded program can only be executed in order. It don't respond to other codes' requests before it hasn't finished the current task. Nevertheless multi-thread technology can realize above-mentioned functions such as pausing, continuing and scraming measurement. The main program is as follows:

$/ *$ create thread pool and new thread*/

CmtNewThreadPool (5, \&poolHandle);

CmtScheduleThreadPoolFunction

(poolHandle, ThreadTest, NULL, \&continueID);

$/ *$ the new thread runs $* /$

Int CVICALLBACK ThreadTest (void *functionData)

$\{\quad \cdots . . . \cdots \cdot / /$ codes of the new thread's callback function $\}$

$/ *$ release the new thread and thread pool*/

CmtWaitForThreadPoolFunctionCompletion(poolHandle, continueID, 0);

CmtReleaseThreadPoolFunctionID(poolHandle, continueID);

CmtDiscardThreadPool(poolHandle);

\subsubsection{Design of Automatic Power-off Function}

After the measurement, the equipment operators often forget to power off the measurement equipment because of operating habit or neglect, which can make the measurement 
equipment power on for a long time, and shorten the lives of equipment 's internal electronic parts. In order to solve this problem, automatic power-off function, which is enabled after the equipment has stopped measuring for some time (can be set programatically), is designed based on asynchronism timer technology in the UI.

Asynchronism timer has been added in LabWindows/CVI 6.0 and later version. Compared to synchronous timer, asynchronous timer runs in a separate thread, can avoid effectively time delay from the main thread and current operations of UI, to avoid the real-time response being destroyed [12]. The main program is as follows:

$/ *$ create a new asynchronous timer */

timerid=NewAsyncTimer ((minute*60), $-1,1$, asynCB, 0);

/* asynchronous timer's callback function */

int CVICALLBACK asynCB (int reserved, int timerId, int event, void *callbackData, int eventData 1 , int eventData 2 )

\{ $\cdots . . . . . . . / /$ codes of the asynchronous timer's callback function $\}$

/ release the new asynchronous timer

DiscardAsyncTimer (timerid);

\subsubsection{Design of Recording and Saving Measurement Re- sults}

The measurement results are recorded and saved in Word format files based on ActiveX technology in UI system. LabWindows/CVI has many standard library functions and software developer kits. For Word files' operation, LabWindows/CVI provides appropriable instrument driver files: word2000.fp and wordreport.fp. The aforementioned two drive files are loaded in the user project, which can correlate Word software to user UI [13]. The main program is as follows:

$/ *$ create and open a new word document*/

WordRpt_ApplicationNew (VFALSE, \&appHandle);

WordRpt_DocumentOpen (appHandle, filename,\&docHandle);

/* data manipulation*/

WordRpt_GoToBookmark(docHandle,"shuqian1");

WordRpt_AppendText(docHandle,Temp);

WordRpt_AddTable(docHandle,rowNum,8, \& tableHandle);

/* Word document's saving and printing*/

WordRpt_DocumentSaveAs (docHandle, filename);

/* shut down Word document*/

CA_DiscardObjHandle (docHandle);

ShutdownWord(\&appHandle, NULL);

\begin{tabular}{|c|c|}
\hline \multicolumn{2}{|c|}{ Test Result } \\
\hline Ho. & Measured Value $(\mathrm{m} \Omega)$ \\
\hline 8 & 218.67 \\
\hline 9 & 211.04 \\
\hline 10 & 213.86 \\
\hline 11 & 222.39 \\
\hline 12 & 8742770.67 \\
\hline 13 & 216.92 \\
\hline 14 & 215.64 \\
\hline 15 & 222.80 \\
\hline 16 & 218.79 \\
\hline
\end{tabular}

Fig. (6). Measurement results of connecting line broken.

\begin{tabular}{|c|c|}
\hline \multicolumn{2}{|c|}{ Test Result } \\
\hline Ho. & Measured Value $(\mathrm{m} \Omega)$ \\
\hline 1 & 410.78 \\
\hline 2 & 397.43 \\
\hline 3 & 409.85 \\
\hline 4 & 402.12 \\
\hline 5 & 10.36 \\
\hline 6 & 397.23 \\
\hline 7 & 412.24 \\
\hline 8 & 403.66 \\
\hline 9 & 396.05 \\
\hline
\end{tabular}

Fig. (7). Measurement results of short-circuiting between two commutator segments.

\section{MEASUREMENT RESULTS AND ANALYSIS}

After the measurement equipment has been designed and manufactured completely, a batch of user-supplied rotor samples are measured, and among one group measurement results is as shown in Fig. (6). Normally, the resistance values between every two adjacent commutator segments should have a good identity, every value's deviation should be less than (below) $\pm 5 \%$ compare with the average value. But the NO.12 measurement value is much larger than others in Fig. (6). Technicians examined and found that the connecting line between one of this group commutator segments and its winding has broken. The whole batch rotors have been measured and short-circuiting between every two adjacent commutator segments hasn't been found. In order to test rotor short-circuiting characteristic, two adjacent commutator segments of one intact rotor are welded together artificially and are marked as NO.5. The measurement results show that the NO.5 measurement value is much less than others as shown in Fig. (7). The experiment shows that short-circuit fault is identified by the measurement equipment. 


\section{CONCLUSION}

An automatic measurement equipment used to measure resistances of between two adjacent commutator segments of motor rotor is designed. The equipment consists on industrial compute, stepping motor drive transmission system, low resistance measuring meter, host compute software. After the assembly, debugging and field trial operation, the euipment has been checked and accepted by user and is regarded as working well. The measurement results indicate that the euipment can measure almost all kind of DC motor rotors accurately, also can record and save measurement results automatically. The euipment uses industrial compute as the control core, controls peripheral hareware units with general purpose interface by programming in the host computer. This design method is applied universally, so it can provide reference and inspiration for other measurement equipments.

\section{CONFLICT OF INTEREST}

The author confirms that this article content has no conflict of interest.

\section{ACKNOWLEDGEMENTS}

This work is supported by the Key Scientific Research Project of Colleges and Universities of Henan Province (No.15A510023 and No.15A413013), the Youth Science Foundation of Henan University of Science and Technology (No.2015QN025) and the High Level Project Cultivation Fund of Henan University of Science and Technology (No. 2015GJB013).

\section{REFERENCES}

[1] Wang Hong-juan, "Usual Trouble Analysis of DC Motors," in MECHANICAL MANAGEMET AND DEVELOPMENT, no.5, pp.32-33, 2003.
[2] Zhang Jian-hui, SONG Ping-gang, "Development of automatic detection system of motor commutating segment based on image recognition technology," in Advanced Technology of ElectricaI Engineering and Energy, vol.24, pp.68-71, 2005.

[3] Hesson Mihyar, "State of the Art End-Of-Line Tester, " in Proceedings of the International Conference on Software Engineering Research and Practise, vol.2, pp.644-649, 2003.

[4] Lita Ioan, Visan Daniel Alexandru, Cioc Ion Bogdan, "Virtual instrumentation application for vibration analysis in electrical equipments testing," in ISSE 2010 - 33rd International Spring Seminar on Electronics Technology: Polymer Electronics and Nanotechnologies: Towards System Integration - Conference Proceedings, 2010,pp.216-219.

[5] Zheng Yongjun, Ma Chao, Zhang Yanchao, Li, Yongchong, Yan, Haijun, "Detection system of droplet impact force based on PVDF sensor, " in Nongye Jixie Xuebao/Transactions of the Chinese Society for Agricultural Machinery, vol.45, pp.142-147, Nov.2014.

[6] LI Zuo-liang, Wang Zuo-quan, "The Measurement Method of Resistances with Digital Multimeter, " in Metrology \& Measurement Technique, vol. 38, no. 11, pp. 29-31, 2011.

[7] Pruitt Beth L.,Kenny Thomas W., "Piezoresistive cantilevers and measurement system for characterizing low force electrical contacts, " in Sensors and Actuators, A: Physical, vol.104, no.1, pp.6877, Mar.2003.

[8] Cimino Charles, "Organic LEDs and how to test them, " in Information Display, vol. 19, no. 11, pp. 28-31, Nov.2003.

[9] Caers J.F.J.M., Zhao X.J., Sy Hansen G., Wong E.H., Mhaisalkar, S.G., "Towards a predictive behavior of non-conductive adhesive interconnects in moisture environment, "In: Proceedings - Electronic Components and Technology Conference, vol. 1, pp. 106112, 2004.

[10] Sheffer Tee, Lantz Paul, "The art of measuring low resistance, " In: EE: Evaluation Engineering, vol. 45, no. 11, pp. 40-46, Nov.2006.

[11] Harrison R.G., Rogers G.W., "Fine wire resistance thermometer amplifier for atmospheric measurementse, " In: Review of Scientific Instruments, vol.77, no.11, 2006.

[12] Ren Xiaojun, Zhou Yu, Xi wenjun, "Design Methods of Multithreading Test and Control Software Based on Labwindows / CVI Platform, " In: Electronic Engineer, vol.32, no.1, pp.5-8, 2006.

[13] Yang Heng-hui, Wang Chao, "Report Technology Based on LabWindows/CVl, "In: Science Technology and Engineering, vol. 11, no. 6, pp.1371-1374, 2011.

Received: October 16, 2014

Revised: December 23, 2014

Accepted: December 31, 2014

(C) Yong et al.; Licensee Bentham Open.

This is an open access article licensed under the terms of the Creative Commons Attribution Non-Commercial License (http://creativecommons.org/licenses/by$\mathrm{nc} / 3.0 /$ ) which permits unrestricted, non-commercial use, distribution and reproduction in any medium, provided the work is properly cited. 